\title{
Industria y empresas en la Argentina, siglo XX (presentación)
}

La historiografía sobre el sector industrial en la Argentina tiene ya una larga tradición aunque no es abundante. Destacan algunas obras generales que han pretendido presentar una visión de largo plazo del desarrollo manufacturero local; los trabajos de Adolfo Dorfman, Aldo Ferrer, Jorge Katz y Bernardo Kosacoff, y Jorge Schvarzer representan hitos de indudable valor, independientemente de sus enfoques de análisis no necesariamente convergentes. ${ }^{1}$

Con todo, resulta notoria la falta de interés por la problemática industrial a partir de mediados de los años setenta y hasta prácticamente el cambio de siglo. Ello fue consecuencia, en parte, de la crisis de los paradigmas estructurales y del cuestionamiento de su valor explicativo, y, en parte del mismo proceso de desindustrialización que se manifestaba en la Argentina y otros países latinoamericanos. La escasa institucionalización académica de esa área de estudios ha sido quizás también uno de los factores que impidieron un mayor desarrollo.

En la última década una serie de investigaciones históricas ha logrado reinsertar la discusión sobre las características que asumió el sector manufacturero principalmente durante el período denominado "industrialización por sustitución de importaciones"

1 Dorfman, Adolfo. Historia de la industria argentina. Buenos Aires: Solar, 1970; Ferrer, Aldo. El devenir de una ilusión. Buenos Aires: Sudamericana, 1989; Katz, Jorge y Kosacoff, Bernardo. El proceso de industrialización en la Argentina. Buenos Aires: CEAL, 1989; Schvarzer, Jorge. La industria que supimos conseguir. Buenos Aires: Planeta, 1996.

Estudos Ibero-Americanos, PUCRS, v. XXXIV, n. 2, p. 7-12, dezembro 2008 
(ISI), entre 1930 y 1976. Esta renovación historiográfica ha permitido focalizar en períodos específicos y contribuir desde nuevos relevamientos empíricos a matizar y, en algunos casos, a rebatir parte de las consideraciones más generales que sobre el sector habían brindado las interpretaciones estructurales o de largo plazo. En efecto, novedosos estudios sobre la industria e instrumentos de la política industrial durante el peronismo llamaron la atención sobre lo escaso que era el conocimiento sobre uno de los momentos más emblemáticos de la política argentina, identificado con la "era de las manufacturas". ${ }^{2}$ Otros períodos, en particular la maduración de la ISI hacia los años sesenta y primeros setenta, han sido menos "revisados", no obstante allí también despuntan algunas indagaciones promisorias. Por ejemplo, recientemente se han abordado los mecanismos que la intervención estatal tuvo para promover grandes emprendimientos industriales que se ubicarían en la cúpula de la elite empresaria local, si bien esta era una línea de investigación ya presente en los años ochenta y noventa. ${ }^{3}$

Más descuidado ha resultado el estudio del sector manufacturero para el período previo al surgimiento de políticas industriales en la década de 1940, precisamente sobre la etapa en la que cierta renovación de los años setenta había indagado más. No obstante, nuevas líneas de investigación pueden encontrarse en los últimos años, referidas a problemáticas novedosas como el consumo, el mercado de capitales o vinculadas a temáticas ya antiguas como la inversión extranjera en el sector. ${ }^{4}$

Pese a estos avances, la carencia de análisis sectoriales es significativa en la literatura especializada argentina. Prácticamente no hay estudios de largo plazo sobre ramas o actividades específicas que permitan una revisión desde esa perspectiva de las pro-

2 Véase por ejemplo Rougier, Marcelo. La politica crediticia del Banco Industrial durante el peronismo. Buenos Aires: CEEED, 2001; Girbal-Blacha, Noemí. Mitos, paradojas y realidades en la Argentina peronista (1946-1955). Bernal: UnQui, 2004; y Belini, Claudio. La industria durante el primer peronismo (1946-1955). Tesis de doctorado, Buenos Aires: UBA, 2004.

3 Por ejemplo, Rougier, Marcelo. Industria, finanzas e instituciones. La experiencia del Banco Nacional de Desarrollo. Bernal: UnQui, 2004; y Castellani, Ana. La ampliación del complejo económico estatal-privado y su incidencia sobre el perfil de la cúpula empresaria. Argentina 1966-1975. H-industri@, año 2, n. 2, primer semestre, Buenos Aires, 2008.

4 Véase, entre otros, Rocchi, Fernando. Consumir es un placer: la industria y la expansión de la demanda a la vuelta del siglo pasado. Desarrollo Económico, n. 148, enero-marzo de 1998; y Pineda, Yovanna. Sources of Finance and Reputation. Merchants Finance Groups in Argentine Industrialization, 1890-1930. Latin American Research Review, n. 2, 2006. 
blemáticas más generales de la industria. Significativamente, la renovación más importante del campo de la historia de la industria en la Argentina ha provenido desde la historiografía de empresas. En efecto, la efervescencia de esta disciplina en los últimos años ha permitido un notable enriquecimiento de los enfoques y de los saberes heredados respecto al sector industrial. Dado que la historia de empresas se focalizó principalmente en compañías y conglomerados manufactureros, el análisis micro permitió incluir temas relacionados a las estrategias empresariales en torno a la incorporación de tecnología, la necesidad de integración, el desarrollo de una red de proveedores o la capacitación de mano de obra, independientemente de los mecanismos específicos de gestión. ${ }^{5} \mathrm{La}$ perspectiva también insufló nuevos aires para la revisión de las políticas estatales respecto al sector, de la importancia de los entornos macroeconómicos y de las relaciones intrasectoriales. ${ }^{6}$

Este dossier se propone contribuir al estudio del sector manufacturero argentino desde las dos perspectivas mencionadas. Por una lado presentamos algunos estudios sectoriales que prestan atención a su vez a la dinámicas empresariales; y por otro un conjunto de estudios que focalizan en las trayectorias de empresas industriales pero que no descuidan su inserción dentro del conjunto de la rama o actividad empresarial; ambas dimensiones de análisis se entrelazan con las políticas industriales desarrolladas. La selección de estos estudios no es caprichosa; ellos cubren, un espacio temporal amplio, el transcurso del siglo $\mathrm{XX}, \mathrm{y}$ variadas experiencias del sector manufacturero (algunas actividades "vegetativas", características de los primeros años de industrialización, y otras "dinámicas", propias de la maduración del sector) con el propósito de aportar a una visión más estilizada de la trayectoria del conjunto.

Los primeros tres trabajos recorren actividades tradicionales de la industria argentina en las primera mitad del siglo XX. Las artes gráficas, la industria textil y la producción tabacalera consti-

5 Véanse principalmente Kosacoff, Bernardo, y otros. Globalizar desde Latinoamérica. El caso Arcor. Bogotá: Mc Graw Hill, 2001; Gilbert, Jorge. Empresario y empresa en la Argentina Moderna. El Grupo Tornquist, 1873-1930. DT n. 27, Buenos Aires: UDESA; Castro, Claudio. Política industria y empresa. El fracaso de Propulsora como polo siderúrgico integrado, 1958-1976. Anuario del CEH "Profesor Carlos Segreti", n. 5, Córdoba, 2005; Rougier, Marcelo y Schvarzer, Jorge. Las grandes empresas no mueren de pie. El (o)caso de SIAM. Buenos Aires: Norma, 2006.

6 Véanse como ejemplo los trabajos incluidos en Rougier, Marcelo (dir.). Políticas de promoción y estrategias empresariales en la Argentina. Buenos Aires: Ediciones Cooperativas, 2007. 
tuyen sectores de larga trayectoria y pueden considerarse "pioneros" en el avance de la sustitución de importaciones, aún durante la vigencia del modelo de extraversión económica que predominó hasta los años treinta.

El artículo de Silvia Badoza analiza el proceso de integración de la Compañía General de Fósforos, una de las más grandes empresas locales en las primeras décadas del siglo XX. Esta empresa avanzó en su proceso de integración hacia la actividad gráfica inicialmente con el propósito de garantizar la provisión de insumos a su producción principal. La autora presenta un estudio de la rama industrial de artes gráficas primero para focalizarse luego en las estrategias empresariales que tendieron a una integración vertical y a la diversificación productiva. El análisis de los ciclos de inversiones permite identificar una fuerte apuesta productiva con el propósito de adquirir un lugar preponderante en el mercado gráfico, una estrategia que resultó exitosa y abrió toda una nueva línea de oportunidades de negocios.

Claudio Belini nos presenta un estudio del sector textil para un período clave en la historia de la industria argentina que comprende la década de 1920 y los primeros años de la Gran Depresión. Discutiendo algunas de las hipótesis más tradicionales sobre el sector, el autor sostiene que antes de los años treinta se observaron progresos en la sustitución de importaciones. Esos avances fueron notorios tanto en la industria lanera como en la fabricación de tejidos de seda artificial, que logró abastecer niveles significativos de la demanda interna. El análisis de la evolución de la rama se completa con un estudio de los principales instrumentos de política económica que afectaron el desempeño de esa industria, en particular del régimen de tarifas aduanero.

El estudio de Noemí Girbal-Blacha aborda también un sector de consumo masivo y tradicional de la industria argentina, pero significativamente muy poco investigado. La autora entrelaza el análisis del sector con las políticas estatales de protección y las características del mercado; allí destacaban unas pocas grandes empresas y una gran cantidad de productores de materia prima, cuyas demandas frente a los poderes públicos no eran necesariamente coincidentes. El análisis de la industria tabacalera en la Argentina, antes y después de la crisis de los años treinta, permite además incorporar la dimensión regional, descuidada en la literatura especializada, salvo las honrosas excepciones de la producción azucarera o vitivinícola. 
Los cuatro trabajos siguientes avanzan temporalmente sobre la última mitad del siglo $\mathrm{XX}$, y recorren actividades industriales vinculadas a lo que se ha denominado la "segunda fase" del proceso ISI. En estos trabajos las políticas públicas de promoción, que han desbordado ya sobradamente la aplicación de pautas arancelarias, adquieren especial relevancia para entender el desempeño productivo de sectores manufactureros enteros y de algunas empresas en particular. Algunos sectores productivos más complejos como la siderurgia, automotriz, química y petroquímica son analizadas aquí combinando la perspectiva de la rama con los estudios de empresas y de las políticas estatales.

El estudio de Marcelo Rougier focaliza en el desempeño productivo de SIAT una empresa del grupo Di Tella creada para fabricar tubos de acero durante los años peronistas. El autor analiza cómo las demandas del sector público en el área de infraestructura estimularon la instalación de la planta y condicionaron luego las estrategias empresariales. El desempeño productivo y el proceso de diversificación que ensaya la firma a partir de los años cincuenta constituyen un resultado de ese vínculo estrecho con el estado que, a su vez, permitió un significativo avance de la sustitución de importaciones en rubros básicos y más intensivos en capital. Adicionalmente, el trabajo presenta información que permite avanzar hacia una mayor comprensión de las políticas industriales durante el peronismo.

Valeria Ianni aborda la instalación y desarrollo de las empresas transnacionales que protagonizaron la fuerte expansión de la rama automotriz en Argentina a comienzo de los años sesenta. La autora destaca el desarrollo de tramas productivas y los eslabonamientos multiplicadores sobre otros sectores industriales de esa implantación, así como una modernización del proceso productivo y de gestión que esas empresas provocaron sobre la estructura industrial local. No obstante, también señala los límites de la experiencia que, en palabras de Ianni, reprodujo el desarrollo desigual en lugar de lograr homogeneizar las condiciones de producción. Un proceso que en definitiva estaba condicionado fuertemente por las estrategias de las empresas transnacionales y dejaba escaso margen de acción a las políticas de promoción locales.

El artículo de Juan Odisio explora un caso específico de promoción industrial en el segundo lustro de los años setenta, cuando el estado, a través de distintos mecanismos, pretendió impulsar algunos grandes proyectos en la producción de insumos 
básicos, a la vez que favorecer la desconcentración regional. El trabajo estudia en particular la conformación del complejo industrial de Petroquímica Bahía Blanca y desentraña el entramado específico que permitió, luego de numerosos avatares, la consolidación de ese polo industrial en el sur de la Provincia de Buenos Aires. Odisio destaca, asimismo, los problemas que tuvo el estado en el desarrollo industrial, tanto en su faz empresarial como en la de promotor del surgimiento y consolidación de empresas privadas.

Finalmente, el trabajo de Graciela Pampin también recala en un caso particular de promoción industrial y regional, aunque mucho menos exitoso que el anterior. Se trata de la empresa Alpat SA, destinada a producir soda solvay, un insumo clave para vastos sectores industriales. El proyecto fue promovido por el estado a fines de los años sesenta y encarado por empresarios privados con fuerte asistencia crediticia pública. Sin embargo, jaqueada por los cambiantes marcos institucionales y legales, la empresa no entró en producción hasta entrado el siglo XXI. El caso ilustra no sólo la falta de coherencia del sector público a la hora de impulsar un proyecto industrial, si no también el nocivo comportamiento empresarial en esas condiciones.

Si bien la fragmentación y la heterogeneidad predominan aun en los estudios industriales y empresariales en la Argentina, el resultado de estas investigaciones que se presentan en conjunto es sin duda alentador. No sólo porque se trata de estudios novedosos en sectores y espacios temporales poco explorados, sino porque sus aportes permiten rediscutir varias de las hipótesis más tradicionales heredades de la literatura especializada. La incipiente renovación de la historia industrial, que se evidencia también en la profusa utilización de fuentes primarias y secundarias prácticamente ausente en los estudios generales, alienta a esperar prontamente nuevas síntesis superadoras en este campo historiográfico.

MARCELO ROUGIER

Recebido em 22 de julho de 2008. Aprovado em 2 de setembro de 2008. 